\title{
Die Umsetzung der nationalen Strategie zur biologischen Vielfalt ist kein Selbstlauf
}

\author{
Jonna Küchler-Krischun · Birgit Mohaupt-Jahr
}

Erhalten: 30. Juli 2008/Akzeptiert: 12. August 2008/Online veröffentlicht: 29. Oktober 2008

(C) Springer-Verlag 2008

Das Bundesministerium für Umwelt, Naturschutz und Reaktorsicherheit (BMU) hat den Umsetzungsprozess zur nationalen Strategie zur biologischen Vielfalt am 5. und 6. Dezember 2007 in Berlin auf dem ersten nationalen Forum zur biologischen Vielfalt mit einer Öffentlichkeitsphase gestartet. In dieser Phase, die auch das erste Halbjahr in 2008 umfasste, sollte die Strategie in ganz Deutschland bekannt gemacht und die verschiedenen gesellschaftlichen Akteure für die Mitwirkung am Umsetzungsprozess eingeladen werden.

Mit den konkreten und messbaren Zielen der Strategie hat die Bundesregierung ein anspruchsvolles Programm beschlossen, das nun konsequent umgesetzt werden muss. Die Umsetzung der nationalen Strategie zur biologischen Vielfalt ist keine Aufgabe für den Bund allein. Die Biodiversitätsstrategie ist eine gesamtgesellschaftliche Strategie, für deren erfolgreiche Umsetzung alle relevanten Akteure aus Politik, Verwaltung und Gesellschaft zusammenarbeiten müssen. Die Öffentlichkeitsphase zählt zu einem mehrjährigen, dialogorientierten Umsetzungsprozess. Bausteine dieses Prozesses sind große nationale und regionale Foren zur biologischen Vielfalt sowie verschiedene akteursspezifische Workshops. Es sind alle staatlichen und nicht-staatlichen Akteure eingeladen, sich am Umsetzungsprozess zu beteiligen.

J. Küchler-Krischun

Bundesministerium für Umwelt, Naturschutz und Reaktorsicherheit

Robert-Schuman-Platz 3, 53175 Bonn, Deutschland

E-Mail: Jonna.Kuechler-Krischun@bmu.bund.de

B. Mohaupt-Jahr $(\bowtie)$

Umweltbundesamt

Wörlitzer Platz 1, 06844 Dessau, Deutschland

E-Mail: Birgit.Mohaupt@uba.de
Dem ersten nationalen Forum zur biologischen Vielfalt folgten sieben regionale Foren im Jahr 2008, bei denen folgende, zentrale Themen der Strategie herausgegriffen und mit Blick auf ihre Umsetzung diskutiert werden:

- Biodiversität und Klimawandel (Januar 2008),

- Biodiversität im urbanen Raum (März 2008),

- Biodiversität, Innovation und naturverträgliches Wirtschaften (April 2008),

- Küsten und marine Biodiversität (April 2008),

- Biodiversität und Wildnis (Mai 2008),

- Biodiversität im ländlichen Raum und naturverträgliche Regionalentwicklung (Juni 2008),

- Internationale Dimension der nationalen Strategie zur biologischen Vielfalt (Juni 2008).

Informationen zu diesen regionalen Foren finden Sie unter: http://www.biologischevielfalt.de. In den Foren hatten die Akteure Gelegenheit, ihre Wünsche und Anregungen zum Umsetzungsprozess der Strategie mitzuteilen und darzustellen, mit welchen Projekten und Initiativen sie selbst die nationale Biodiversitätsstrategie unterstützen wollen. Nach und nach werden alle Kurzdokumentationen der Ergebnisse der Foren auf der Internetseite eingestellt. Es wird auch eine Auswertung aller Hinweise und Anregungen aus dem bisherigen Dialogprozess erarbeitet. Die daraus vom BMU gezogenen Schlussfolgerungen für den Umsetzungsprozess werden zusammen mit den Akteuren beim 2. Nationalen Forum im Januar 2009 diskutiert werden.

In der nationalen Strategie zur biologischen Vielfalt sind ganz konkrete, quantifizierte Ziele (insgesamt rund 330) festgehalten, die bis zu einem bestimmten Zeitpunkt erreicht sein sollen. Dadurch wird auch der Erfolg des Umsetzungsprozesses direkt kontrollierbar. Die Bundesregierung hat sich selbst zur Erfolgskontrolle verpflichtet, indem sie in jeder Legislaturperiode einen umfassenden Bericht über die Erreichung der Ziele vorlegen wird. Insgesamt muss aber 
national wie international noch mehr getan werden und vor allem die Zusammenarbeit zwischen den einzelnen Akteuren deutlich verbessert werden.

Das Thema hat zwar im Vorfeld der UN-Naturschutzkonferenz im Mai 2008 in Bonn deutlich an politischer Relevanz und öffentlicher Akzeptanz gewonnen. Jedoch ist auch weiterhin starker Aufklärungsbedarf notwendig, welche
Konsequenzen der immer noch nicht gebremste Verlust an Biodiversität für künftige Generationen bedeutet.

\section{Literatur}

Nationale Strategie der biologischen Vielfalt der Bundesrepublik Deutschland. Berlin, 2007, www.biologischevielfalt.de 